\title{
Neural Correlates of Distinct Cognitive Phenotypes in Early Parkinson's Disease
}

\author{
Ece Bayram ${ }^{1}$, Brent Bluett ${ }^{2}$, Xiaowei Zhuang ${ }^{1}$, Dietmar Cordes ${ }^{1}$, Denise R. LaBelle ${ }^{1}$, and \\ Sarah J. Banks ${ }^{3}$ \\ ${ }^{1}$ Cleveland Clinic Lou Ruvo Center for Brain Health, Las Vegas, Nevada, USA. \\ 2Stanford University, Department of Neurology and Neurological Sciences, Palo Alto, California, \\ USA.
}

${ }^{3}$ University of California San Diego, Department of Neuroscience, La Jolla, California, USA.

\section{Abstract}

Objective: Cognitive decline is common in Parkinson's disease (PD), but changes can occur in a variety of cognitive domains. The lack of a single cognitive phenotype complicates diagnosis and tracking. In an earlier study we used a data-driven approach to identify distinct cognitive phenotypes of early PD. Here we identify the morphometric brain differences between those different phenotypes compared with cognitively normal PD participants.

Methods: Six different cognitive classes were included (Weak, Typical, Weak-Visuospatial/ Strong-Memory, Weak-Visuospatial, Amnestic, Strong). Structural differences between each class and the Typical class were assessed by deformation-based morphometry.

Results: The different groups evidenced different patterns of atrophy. Weak class had frontotemporal and insular atrophy; Weak-Visuospatial/Strong-Memory class had frontotemporal, insular, parietal, and putamen atrophy; Weak-Visuospatial class had Rolandic operculum; Amnestic class had left frontotemporal, occipital, parietal and insular atrophy when compared to the Typical class. The Strong class did not have any atrophy but had significant differences in left temporal cortex in comparison to the Typical class.

Conclusions: Structural neuroimaging differences are evident in PD patients with distinct cognitive phenotypes even very early in the disease process prior to the emergence of frank cognitive impairment. Future studies will elucidate whether these have prognostic value in identifying trajectories toward dementia, or if they represent groups sensitive to different treatments.

Corresponding author: Ece Bayram. Corresponding author at: Cleveland Clinic Lou Ruvo Center for Brain Health, 888 West Bonneville Avenue, Las Vegas, NV 89106, USA.

Publisher's Disclaimer: This is a PDF file of an unedited manuscript that has been accepted for publication. As a service to our customers we are providing this early version of the manuscript. The manuscript will undergo copyediting, typesetting, and review of the resulting proof before it is published in its final citable form. Please note that during the production process errors may be discovered which could affect the content, and all legal disclaimers that apply to the journal pertain.

7. Declarations of interest:

None for Drs. Bayram, Zhuang, Cordes, LaBelle and Banks.

Dr. Bluett has received speaking and consulting fees from Abbvie, and speaking fees from Teva pharmaceutical. 


\section{Keywords}

Cognitive dysfunction; Cluster analysis; Parkinson's disease; Neuroimaging; Magnetic resonance imaging; atrophy

\section{Introduction}

Parkinson's disease (PD) is a neurodegenerative disorder characterized by motor and nonmotor impairments. Cognitive impairments are quite heterogeneous and can be observed during all stages of the disease, including the prodromal phase. Executive, visuospatial, and memory deficits are common, [1] and up to $80 \%$ of patients develop dementia [2]. However, the underlying neuroanatomical mechanisms of cognitive deficits in PD have yet to be clarified, especially early in the disease course when cognitive changes, if present, are subtle. Establishing underlying structural changes of cognitive impairments may be important for the early detection and management of these impairments, as well as prognosis.

Several cortical and subcortical regions have been implicated in cognitive impairments in PD. PD patients with dementia (PDD) and mild cognitive impairment (PD-MCI) have frontal, temporal, and parietal atrophy [3-7]. Atrophy to subcortical structures including the thalamus, putamen, hippocampus, amygdala, and caudate have also been reported [3,8-11]. However, results vary greatly in the studies of PD-MCI possibly due to the heterogeneity of PD-MCI, or specific to how MCI was defined and assessed in each study. For example, one study reported atrophy in only subcortical regions [8], while another study showed no structural differences in PD-MCI compared to healthy controls [12]. Another study demonstrated insular, frontal, and temporal atrophy only in the left hemisphere in PD-MCI compared to cognitively normal PD [13]. Additionally, studies investigating different cognitive domains suggest that changes in distinct regions are involved in each domain in PD. Memory performance was related to medial temporal lobe structure volumes, whereas visuospatial deficits have been attributed to superior temporal, parietal, and occipital volume loss [14-17]. Further classification of PD-MCI based on affected cognitive domains may provide more consistent results in future studies.

These structural changes can also present early on in the disease alongside cognitive deficits. In early PD, memory deficits have been associated with cortical thinning in occipital, superior parietal and postcentral gyri [18]. In a study focusing on de novo PD, PD-MCI group had verbal memory deficits and temporal lobe, including the hippocampus; and parietal lobe with the precuneus and posterior cingulate gyrus atrophy compared to cognitively intact PD patients [19]. More interestingly, these changes can be seen even without pronounced cognitive deficits in PD. In a sample of generally cognitively intact PD patients, weaknesses in the cognitive profile were associated with distinct anatomical patterns: deficits in executive functioning were associated with decreased volumes within regions of the frontostriatal cognitive-control network, lower memory scores were associated with decreased volume in frontostriatal cognitive-control network regions and the medial 
temporal lobe, and worse visuospatial performance was associated with temporo-occipital volume reduction [14].

In a previous study, we used latent class analysis (LCA) to identify different cognitive phenotypes in early PD [20]. This technique is purely data-driven, not requiring a priori hypotheses, and extracts classes based on patterns of cognitive scores between patients. Based on LCA, several classes emerged including the cognitively strong, those with isolated memory, and visuospatial weaknesses. Demographics and disease characteristics were evaluated only after the classes were created. Cognitive performance was independent of motor and non-motor impairments, cerebrospinal fluid (CSF) biomarkers, and striatal dopamine transporter (DaT) binding ratios. In this study, we investigated the differences in structural integrity between these classes to determine underlying neuroanatomical alterations of PD patients with different cognitive profiles. Due to multiple networks and structures being implicated in cognitive impairment in PD [21,22], instead of regional volume analysis, a whole brain analysis approach without a priori determined regions of interest was implemented. We utilized deformation based morphometry (DBM), as this technique preserves the entirety of the imaging data and has a higher sensitivity for subcortical atrophy, increasing the likelihood of detecting early changes in de novo PD [23].

\section{Material and Methods}

\subsection{Participants}

122 de novo, medication-naïve PD subjects with 3 T baseline MRI from the PPMI database were included. Details of the aims and design of the PPMI have been published elsewhere [24] and can be found on the PPMI website (http://www.ppmi-info.org/study-design). The study was approved by the institutional review board at each site and all participants provided written informed consent. The research was completed in accordance with Helsinki Declaration.

\subsection{Cognitive measures and classes}

Classes reported in our previous study were used [20]. Cognitive tests included Judgment of Line Orientation (JLO), learning and recall scores of the Hopkins Verbal Learning TestRevised (HVLT-R), Letter Number Sequencing (LNS), Symbol-Digit Modalities Test (SDMT), and semantic fluency with three prompts (animals, vegetables and fruits). Raw scores were Z-transformed against the mean of all cases. These scores served as cluster indicators. Clusters of variables were identified and labeled based on the characteristics of the variables within each cluster. Then clusters were compared to each other. The solution with the lowest Bayesian Information Criterion statistic, good class stability, and unique performance patterns across classes was selected as the best fit solution. This resulted in six cognitive classes in total; the Weak-Overall, Typical-Overall, Strong-Overall, StrongMemory, Weak-Visuospatial, and Amnestic. The Weak-Overall class demonstrated poor performance across all cognitive measures, the Typical-Overall class had average, and the Strong-Overall class demonstrated strong cognition. The Strong-Memory class performed above average in the memory tests, and had poor visuospatial functioning. The Weak- 
Visuospatial class exhibited an isolated weakness in visuospatial functioning. Finally, the Amnestic class had weaknesses in learning and memory functions.

We relabeled the Weak-Overall class as Weak, Typical-Overall as Typical, and StrongOverall as Strong for ease in this study. To emphasize the visuospatial weakness in the Strong-Memory class, this class was relabeled as Weak-Visuospatial/Strong Memory class. The other class labels were not changed. 7 subjects in the Weak class, 30 in the Typical class, 9 in the Weak-Visuospatial/Strong-Memory, 21 in the Weak-Visuospatial, 26 in the Amnestic, 29 in the Strong class had available imaging data and were included. Although class identification was independent of norms, we further investigated the frequency of MCI for each class. Due to the limited number of cognitive tests included in the PPMI, Movement Disorders Society Task Force Level I criteria was used to screen for MCI [25]. Standard scores were used to determine MCI or high functioning. None of the participants had scores 1.5 standard deviation higher than the normal mean across all tests. However, as individuals in the Strong class performed above normal levels in some of tests $(13.8 \%$ above norms in semantic fluency, 3.4\% in SDMT, 34.5\% in JLO, 24.1\% in LNS, 20.7\% in HVLT-R Learning, $31 \%$ in HVLT-R Recall), the Typical class was used as the reference group.

Disease characteristics were assessed by the Movement Disorders Society-Unified Parkinson's Disease Rating Scale. Disease severity was determined by Hoehn and Yahr staging (HYS). Modified Schwab and England Activity of Daily Life (ADL) was used to assess patients' independency level. Non-motor scales included the Geriatric Depression Scale (GDS), State-Trait Anxiety Inventory (STAI), REM Sleep Behavior Disorder Screening Questionnaire (RBDSQ), Epworth Sleepiness Scale (ESS), University of Pennsylvania Smell Identification Test (UPSIT) and the Scales for Outcomes in Parkinson's Disease-Autonomic (SCOPA-AUT).

Apolipoprotein E e4 (APOEe4) status was included for subjects with available genotyping. CSF biomarkers included amyloid- $\beta$, $\alpha$-synuclein, phosphorylated-tau ( $\mathrm{p}$-tau), and total tau. Striatal DaT binding ratios were derived for the left and right caudate and putamen by single photon emission CT (SPECT) imaging with [123I]FP-CIT radioligand. Binding was calculated by a standardized volume of interest template for each region compared to an occipital reference region.

\subsection{MRI data acquisition}

All imaging data were acquired from the PPMI database. High resolution T1-weighted MRI structural images were collected for the subjects across 5 different scanners. 15 subjects were scanned on a GE Signa 3.0T HDx scanner using the 3D fast SPGR sequence (8 subjects with a voxel size of $1 \times 1 \times 1.2 \mathrm{~mm}^{3}$, matrix size: $256 \times 256,152$ sagittal slices; 7 subjects with a voxel size of $1 \times 1 \times 0.7 \mathrm{~mm}^{3}$, matrix size: $256 \times 256,248$ sagittal slices). 3 subjects were scanned on a GE 3.0T Discovery scanner using the fast SPGR sequence with a voxel size of $1 \times 1 \times 1.2 \mathrm{~mm}^{3}$, matrix size: $256 \times 256$ and 152 sagittal slices. 8 subjects were scanned on a Philips Achieva 3.0T scanner using the MPRAGE sequence with a voxel size of $0.92 \times 0.92 \times 1.2 \mathrm{~mm}^{3}$, matrix size: $288 \times 288$ and 152 sagittal slices (1 subject acquired 152 axial slices). 6 subjects were scanned on a SIEMENS 3.0T Verio scanner using the MPRAGE sequence with a voxel size of $1 \times 1 \times 1 \mathrm{~mm}^{3}$, matrix size $240 \times 256,176$ sagittal 
slices. 90 subjects were scanned on a Siemens 3.0T Trio scanner using the MPRAGE sequence ( 88 subjects with a voxel size of $1 \times 1 \times 1 \mathrm{~mm}^{3}$, matrix size $240 \times 256,176$ sagittal slices; 1 subject with a voxel size of $0.98 \times 0.98 \times 1 \mathrm{~mm}^{3}$, matrix size $256 \times 256,160$ sagittal slices and 1 subject with a voxel size of $1 \times 1 \times 1 \mathrm{~mm}^{3}$, matrix size $240 \times 256,196$ sagittal slices). All T1 images were acquired with the following imaging parameters: TR, $2300 \mathrm{~ms}$; TE, $2.98 \mathrm{~ms}$; flip angle, $9^{\circ}$; and TI, $900 \mathrm{~ms}$.

2.3.1 Deformation based morphometry (DBM)—DBM analysis was performed using the computational anatomy toolbox (CAT) for SPM (http://www.neuro.uni-jena.de/ cat/). T1 images from 122 subjects were first re-sliced to a uniform voxel size of $1 \times 1 \times 1$ $\mathrm{mm}^{3}$ and then preprocessed with signal inhomogeneity correction and global intensity normalization. The preprocessed $\mathrm{T} 1$ image of each subject was then non-linearly normalized to the standard MNI-152 $1.5 \mathrm{~mm}$ template to minimize the regional differences due to local deformations. The type and localization of the structural differences between the subject brain and the template brain were minimized in this non-linear normalization step and coded in the deformation field. The retrospective quality control framework in CAT were performed and all images were scored C- or above, lying in or above the satisfactory category. Percentage change of each voxel volume was finally quantified by the Jacobian determinant of the deformation field [26]. The deformation field map from each subject was further smoothed using an $8 \mathrm{~mm}$ Gaussian kernel before the statistical analysis.

\subsection{Statistical analysis}

Statistical analyses of demographics, disease characteristics and test scores were completed using SPSS V 23.0. Numerical data are reported as the mean (standard deviation) and categorical data as percentage unless stated otherwise. Since not all of the participants from our previous study were included, all variables were reassessed across groups.

Demographics, disease characteristics, and test scores across groups were compared by analysis of variance (ANOVA) with Dunnett's post hoc test (Typical class as the reference) and chi-square tests. $\mathrm{p}<0.05$ was considered statistically significant.

Statistical comparisons for brain atrophy were performed on Jacobian determinants of the deformation field between each cognitive class and the Typical class. The Typical class was chosen for comparison as this group had an average cognitive performance across domains. Two-sample t-tests were performed with age, gender, year of education, scanner type, and handedness as covariates. Statistical significance level was established at family-wise corrected p-value of 0.05 (cluster-wise corrected, $|t|>2.4$ with at least 1589 voxels in the cluster). Covariates were regressed out from the data to determine the effect sizes of regional volume differences. Cohen's d was calculated by dividing the difference between the means of two groups by the pooled standard.

Using the same analysis approach, comparisons between Weak-Visuospatial/Strong-Memory and Amnestic class were done to investigate the structural associates of memory performance (Supplementary Results). 


\section{Results}

\subsection{Demographics, biomarkers and disease characteristics}

Demographics and disease characteristics are summarized in Table 1. Non-motor symptoms and biomarkers are shown in Supplementary Table 1. As all participants were de novo PD, disease severity only ranged between HYS 1 and 2. There were group differences in MDSUPDRS Part I, RBDSQ, SCOPAAUT and MoCA scores. Weak class had higher MDSUPDRS Part I ( $\mathrm{p}=.039)$, RBDSQ ( $\mathrm{p}=.019)$, and SCOPAAUT ( $\mathrm{p}=.024)$ scores compared to the Typical class. None of the cognitive classes differed from the Typical class for the MoCA score.

\subsection{Cognitive classes}

Raw scores on cognitive tests are given in Table 2. All tests were different across the cognitive classes. Comparisons with Typical class showed that Weak class had lower scores on all tests ( $\mathrm{p}=.000$ for all tests) except for semantic fluency. Weak-Visuospatial/StrongMemory class had lower score on JLO and higher scores in HVLT-R learning and recall compared to the Typical class ( $\mathrm{p}=.000$ for all). Weak-Visuospatial class had lower score in JLO compared to the Typical class ( $\mathrm{p}=.000)$. Amnestic class had lower scores in HVLT-R learning and recall compared to the Typical class $(\mathrm{p}=.000)$. Strong class had higher scores in semantic fluency ( $\mathrm{p}=.035)$, LNS ( $\mathrm{p}=.007)$, HVLT-R learning and recall ( $\mathrm{p}=.000$ for both) compared to the Typical class.

\subsection{Deformation based morphometry (DBM) results}

Coordinates of the significantly different clusters in each cognitive class compared to the Typical class are summarized in Table 3. Weak class had the most extensive atrophy including regions of left frontotemporal, insular; and right frontal lobes with a very large mean effect size [Cohen's d=1.24 (0.22)] (Figure 1a). Weak-Visuospatial/Strong-Memory class had left frontotemporal, insular, parietal; right frontal and putamen atrophy with a large mean effect size [Cohen's d=1.00 (0.15)] (Figure 1b). Weak-Visuospatial class only had atrophy in the left Rolandic operculum with a large mean effect size [Cohen's d=0.81 (0.11)] (Figure 1c). Amnestic class had left inferior temporal, occipital; right motor cortex, parietal and insular atrophy with a large mean effect size [Cohen's $d=0.86$ (0.37)] (Figure 1d). Compared to the Strong class, Typical class had atrophy in left middle and inferior temporal gyri with a medium-large mean effect size [Cohen's d=-0.66 (0.06)] (Figure 1e). The only overlap between Weak-Visuospatial/Strong Memory and Weak-Visuospatial class when compared to the Typical class, was the atrophy in left precentral gyrus (Figure 2 illustrates the overlap, not the statistical contrast).

\section{Discussion}

The current study sought to investigate the structural neuroanatomic correlates of different cognitive profiles in recently diagnosed, nondemented PD patients. We compared groups of PD patients with data-driven patterns of cognitive weakness to a comparison group of PD patients with average scores on cognitive tests. We were able to demonstrate subtle structural differences in these classes which might be important in understanding the earliest cognitive 
disruption in PD. As our main concern was to investigate neural correlates of different types of cognitive weakness in PD, but not PD itself as a disorder, we chose not to include a healthy control group. By using a group of PD patients with average cognitive performance as a control group, we aimed to control for the effects of PD (neural correlates of motor or other non-motor changes) and focus solely on cognition. Additionally, we aimed to investigate whether neural correlates of cognitive weakness can be found early in the cognitive impairment process, thus we only included nondemented PD patients.

The Weak class had both the most extensive set of cognitive weaknesses and the most widespread pattern of atrophy. They demonstrated worse executive and visuospatial function, learning and memory scores than the Typical class. The analysis demonstrated atrophy in the lateral and inferior temporal regions, posteromedial and lateral frontal regions, insula and motor cortex in the Weak class. Lateral and inferior temporal regions are associated with semantic processing [27,28]. Posteromedial and lateral frontal regions are suggested to work together for attention and error monitoring [29]. Insula has been associated with sensorimotor processing and general cognition [30]. Lateral temporal and frontal regions, as well as the insula have also been associated with visuospatial functioning using lesion-symptom mapping in ischemic stroke patients [31]. In a previous study volumetric reduction in insular, superior frontal, and lateral temporal grey matter was observed in PD-MCI compared to PD with no cognitive impairment [13]. In this study, insular atrophy was associated with executive function and attention; lateral temporal atrophy was associated with executive function, attention and memory, superior frontal atrophy was associated with attention impairment. Our findings suggest that even in a sample of PD patients who have subtle cognitive weakness as opposed to the frank impairments seen in MCI, structural patterns observed in PD-MCI may be present. While interpreting the findings within this class, it is also important to consider that the sample size was limited to only seven PD patients in this cognitive class.

The Weak-Visuospatial/Strong-Memory class had worse visuospatial functioning coupled with better learning and memory performance compared to the Typical class. Compared to the Typical class, atrophy in prefrontal, lateral temporal, parietal, insular, motor cortex; and subcortical regions was found. Previous studies indicate that frontal, lateral temporal, insula, and basal ganglia volumetry is associated with visuospatial decline [31]. The involvement of putamen in visuospatial function was shown in nondemented PD patients with visuospatial weakness in a previous study [32]. As regions with volumetric reductions we found in this class are in line with previous studies on visuospatial functioning associations of regional grey matter volume, the structural differences between this class and the Typical class are likely to be driven by visuospatial weakness rather than learning and memory strength. However, the small number of patients in this class make it hard to interpret the results.

The only differences between Weak-Visuospatial class and the Typical class were weakness in visuospatial functioning, and atrophy in left rolandic operculum including the precentral gyrus and the insula. In PD, atrophy of the Rolandic operculum has been seen in patients with mild to moderate diseases [33]. In particular, left insular atrophy has been shown to correlate with visuospatial performance in PD-MCI [34]. Our findings indicate that the atrophy pattern in PD-MCI associated with visuospatial functioning present itself early on in 
the disease without any significant visuospatial deficits, but only weaknesses. In addition to volumetry, structural connectivity of insula was also implicated in a visuospatial attention task [35]. Thus, insula has the potential to serve as a marker for future visuospatial decline. More interestingly, this class had less atrophy than Weak-Visuospatial/Strong Memory class when both classes were compared to the Typical class. This suggests that the pathophysiology of the cognitive weaknesses in these two groups are different. The overlap between the regions with atrophy in these two classes may be responsible for the visuospatial weakness seen in both classes. Traditionally known for its involvement in movement control, precentral gyrus also plays a role in motor imagery and spatial transformations [36]. Working memory assessed with recalling sensory materials and movement sequences after a time delay has also been associated with precentral gyrus activation [37]. Precentral gyrus atrophy is also observed in PD-MCI [38], and progression to $\mathrm{MCI}$ in these classes may be associated with atrophy in this region. A longitudinal study focusing on the progression of cognitive weaknesses to deficits in these classes can help clarify this hypothesis.

The Amnestic class significantly differed from the Typical class on learning and memory. Previous studies in early PD reported that atrophy in the medial temporal lobe structures correlate with memory functioning similar to Alzheimer's disease [11,15]. Lateral temporal, parietal, occipital, insular and motor cortex atrophy in this class is in line with the atrophy pattern in PD-MCI [13]. This class is likely to have a higher rate of conversion to MCI, as alongside bradykinesia, years of education, subjective cognitive decline report, REM sleep behavior disorder; verbal memory retention is the only cognitive predictor of future cognitive decline in de novo PD patients [39]. Our results reveal that PD-MCI atrophy patterns are present in patients with weaknesses in only verbal learning and memory. The overlap between atrophic regions in previously reported PD-MCI and the Amnestic class suggests that this cognitive class alongside the Weak class are the two classes most likely to progress to PD-MCI. This should be evaluated longitudinally by characterizing PD patients without any cognitive impairment but rather weaknesses in learning and memory or overall cognitive performance during the baseline. These patients may have a higher risk of developing cognitive impairment more rapidly.

The Strong class performed better on executive function, learning and memory tests; and did not show any atrophy patterns compared to the Typical class. Compared to the Strong class, the Typical class had atrophy in left middle and inferior temporal gyri. These gyri have been associated with several cognitive domains including language, semantic memory, and visual perception [27]. Thus, atrophy in these regions, seen in the Typical class, might lead to difficulty in encoding and then recalling a list of words from certain semantic categories which may easily elicit visual representations in the normal brain. Additionally, structural differences were limited to the left hemisphere. The left hemisphere is more involved with language and motor control in both right-handed and left-handed individuals [40]. Therefore classes identified by scores on both verbal and nonverbal measures may provide different results and should be further investigated in future studies.

One of the major limitations in our study was the restricted number of cognitive tests which were used to determine the cognitive profiles. PPMI is a multi-center study aiming at 
establishing biomarkers for PD, which includes data for multiple features related to PD. While the accessibility of this dataset provides an opportunity to conduct research on large numbers of patients in a readily available dataset, the number of cognitive tests is restricted within each cognitive domain. This potentially limits the reliability of the cognitive profiles included in our study. For example, the Movement Disorders Society Level II criteria for MCI requires at least two independent tests for each domain [25]. Therefore future studies would benefit from including more cognitive tests evaluating each domain with at least two independent tests to establish cognitive classes. Our imaging findings should also be interpreted in the light of this limitation, which limit the reliability of the structural findings. Another limitation is the number of patients included in the groups. There were only seven patients in the Weak class. Although this low number reduces the reliability of the findings within this class, high effect sizes $(|\mathrm{d}|>0.8)$ suggest that the results may reflect a true finding rather than an artefact. In conclusion, our findings indicate that different, data-driven, cognitive profiles in PD are related to specific structural patterns. These patterns can be observed within the first two years of the disease, and before any treatment has been administered. Future studies should include a more comprehensive neurocognitive battery to assess each cognitive domain extensively, using both verbal and nonverbal measures in larger patient samples. Increasing the statistical power may identify a stronger correlation between the cognitive profiles and associated structural changes. Additionally, studies focusing on structural and functional connectivity may help understand the role of specific networks involving these regions in cognitive impairments in PD as the symptoms of this disease are due to disrupted networks rather than atrophy in single regions. Prospective validation of the correlation between the cognitive profiles and underlying structural and functional changes in de-novo PD patients should be performed longitudinally as well. Due to the cross-sectional nature of our study, we cannot know whether these atrophy patterns associated with cognitive classes are independent of PD progression and solely reflect cognitive weaknesses. Future studies evaluating the PD patients with different cognitive phenotypes in a longitudinal manner, can reveal whether specific cognitive deficits are due to the baseline structural differences.

\section{Conclusions}

This study shows that cognitive weaknesses in de novo PD without dementia have distinct atrophy patterns. The atrophy pattern in each cognitive class is not due to the PD but rather to cognitive performance. Latent class analysis determining cognitive class can help establish imaging biomarkers in early PD patients.

\section{Supplementary Material}

Refer to Web version on PubMed Central for supplementary material.

\section{Acknowledgements}

Research reported in this publication was supported by an institutional development award (IDeA) from the National Institute of General Medical Sciences of the National Institutes of Health under grant number 5P20GM109025. Data used in the preparation of this article were obtained from the Parkinson's Progression Markers Initiative (PPMI) database (www.ppmi-info.org/data). For up-to-date information on the study, visit www.ppmi-info.org. PPMI - a public-private partnership - is funded by the Michael J. Fox Foundation for 
Parkinson's Research and funding partners, including AbbVie, Avid Radiopharmaceuticals, Biogen, Bristol-Myers Squibb, Covance, GE Healthcare, Genentech, GlaxoSmithKline (GSK), Eli Lilly and Company, Lundbeck, Merck, Meso Scale Discovery (MSD), Pfizer, Piramal Imaging, Roche, Servier, and UCB (http://www.ppmi-info.org/ fundingpartners). This foundation is responsible for PPMI study design and data collection, but is not involved in data analysis.

\section{References}

[1]. Aarsland D, Cognitive impairment in Parkinson's disease and dementia with Lewy bodies, Parkinsonism Relat. Disord 22 Suppl 1 (2015) S144-8. doi:10.1016/j.parkreldis.2015.09.034. [PubMed: 26411499]

[2]. Aarsland D, Beyer MK, Kurz MW, Dementia in Parkinson's disease, Curr. Opin. Neurol 21 (2008) 676-682. doi:10.1097/WCO.0b013e3283168df0. [PubMed: 18989112]

[3]. Burton EJ, McKeith IG, Burn DJ, Williams ED, O’Brien JT, Cerebral atrophy in Parkinson's disease with and without dementia: A comparison with Alzheimer's disease, dementia with Lewy bodies and controls, Brain. 127 (2004) 791-800. doi:10.1093/brain/awh088. [PubMed: 14749292]

[4]. Beyer MK, Larsen JP, Aarsland D, Gray matter atrophy in Parkinson disease with dementia and dementia with Lewy bodies, Neurology. 69 (2007) 747-754. doi:10.1212/01.wnl. 0000269666.62598.1c. [PubMed: 17709706]

[5]. Melzer TR, Watts R, MacAskill MR, Pitcher TL, Livingston L, Keenan RJ, Dalrymple-Alford JC, Anderson TJ, Grey matter atrophy in cognitively impaired Parkinson's disease, J. Neurol. Neurosurg. Psychiatry 83 (2012) 188-194. doi:10.1136/jnnp-2011-300828. [PubMed: 21890574]

[6]. Song SK, Lee JE, Park HJ, Sohn YH, Lee JD, Lee PH, The pattern of cortical atrophy in patients with Parkinson's disease according to cognitive status, Mov. Disord 26 (2011) 289-296. doi: 10.1002/mds.23477. [PubMed: 21370255]

[7]. Pereira JB, Svenningsson P, Weintraub D, Brønnick K, Lebedev A, Westman E, Aarsland D, Initial cognitive decline is associated with cortical thinning in early Parkinson disease, Neurology. 82 (2014) 2017-2025. doi:10.1212/WNL.0000000000000483. [PubMed: 24808018]

[8]. Apostolova LG, Beyer M, Green AE, Hwang KS, Morra JH, Chou YY, Avedissian C, Aarsland D, Janvin CC, Larsen JP, Cummings JL, Thompson PM, Hippocampal, caudate, and ventricular changes in Parkinson's disease with and without dementia, Mov. Disord 25 (2010) 687-695. doi: 10.1002/mds.22799. [PubMed: 20437538]

[9]. Junqué C, Ramírez-Ruiz B, Tolosa E, Summerfield C, Martí MJ, Pastor P, Gómez-Ansón B, Mercader JM, Amygdalar and hippocampal MRI volumetric reductions in Parkinson's disease with dementia, Mov. Disord 20 (2005) 540-544. doi:10.1002/mds.20371. [PubMed: 15645532]

[10]. Mak E, Bergsland N, Dwyer MG, Zivadinov R, Kandiah N, Subcortical atrophy is associated with cognitive impairment in mild parkinson disease: A combined investigation of volumetric changes, cortical thickness, and vertex-based shape analysis, Am. J. Neuroradiol 35 (2014) 22572264. doi:10.3174/ajnr.A4055. [PubMed: 25082821]

[11]. Weintraub D, Neurodegeneration Across Stages of Cognitive Decline in Parkinson Disease, Arch. Neurol 68 (2011) 1562. doi:10.1001/archneurol.2011.725. [PubMed: 22159053]

[12]. Dalaker TO, Zivadinov R, Larsen JP, Beyer MK, Cox JL, Alves G, Bronnick K, Tysnes OB, Antulov R, Dwyer MG, Aarsland D, Gray matter correlations of cognition in incident Parkinson's disease, Mov. Disord 25 (2010) 629-633. doi:10.1002/mds.22867. [PubMed: 20213820]

[13]. Mak E, Zhou J, Tan LCS, Au WL, Sitoh YY, Kandiah N, Cognitive deficits in mild Parkinson's disease are associated with distinct areas of grey matter atrophy, J. Neurol. Neurosurg. Psychiatry 85 (2014) 576-580. doi:10.1136/jnnp-2013-305805. [PubMed: 24133286]

[14]. Filoteo JV, Reed JD, Litvan I, Harrington DL, Volumetric correlates of cognitive functioning in nondemented patients with Parkinson's disease, Mov. Disord 29 (2014) 360-367. doi:10.1002/ mds.25633. [PubMed: 24038502]

[15]. Beyer MK, Bronnick KS, Hwang KS, Bergsland N, Tysnes OB, Larsen JP, Thompson PM, Somme JH, Apostolova LG, Verbal memory is associated with structural hippocampal changes in 
newly diagnosed Parkinson's disease, J. Neurol. Neurosurg. Psychiatry 84 (2013) 23-28. doi: 10.1136/jnnp-2012-303054. [PubMed: 23154124]

[16]. Bouchard TP, Malykhin N, Martin WRW, Hanstock CC, Emery DJ, Fisher NJ, Camicioli RM, Age and dementia-associated atrophy predominates in the hippocampal head and amygdala in Parkinson's disease, Neurobiol. Aging 29 (2008) 1027-1039. doi:10.1016/j.neurobiolaging. 2007.02.002. [PubMed: 17368653]

[17]. Pereira JB, Junqué C, Martí MJ, Ramirez-Ruiz B, Bargalló N, Tolosa E, Neuroanatomical substrate of visuospatial and visuoperceptual impairment in Parkinson's disease, Mov. Disord 24 (2009) 1193-1199. doi:10.1002/mds.22560. [PubMed: 19412935]

[18]. Uribe C, Segura B, Baggio HC, Abos A, Garcia-Diaz AI, Campabadal A, Marti MJ, Valldeoriola F, Compta Y, Tolosa E, Junque C, Cortical atrophy patterns in early Parkinson's disease patients using hierarchical cluster analysis, Parkinsonism Relat. Disord (2018). doi:10.1016/j.parkreldis. 2018.02.006.

[19]. Noh SW, Han YH, Mun CW, Chung EJ, Kim EG, Ji KH, Seo JH, Kim SJ, Analysis among cognitive profiles and gray matter volume in newly diagnosed Parkinson's disease with mild cognitive impairment, J. Neurol. Sci 347 (2014) 210-213. doi:10.1016/j.jns.2014.09.049. [PubMed: 25451006]

[20]. Labelle DR, Walsh RR, Banks SJ, Latent Cognitive Phenotypes in de Novo Parkinson's Disease: A Person-Centered Approach, J. Int. Neuropsychol. Soc 23 (2017) 551-563. doi:10.1017/ S1355617717000406. [PubMed: 28651678]

[21]. Hattori T, Orimo S, Aoki S, Ito K, Abe O, Amano A, Sato R, Sakai K, Mizusawa H, Cognitive status correlates with white matter alteration in Parkinson's disease, Hum. Brain Mapp. 33 (2012) 727-739. doi:10.1002/hbm.21245. [PubMed: 21495116]

[22]. Chen B, Fan GG, Liu H, Wang S, Changes in anatomical and functional connectivity of Parkinson's disease patients according to cognitive status, Eur. J. Radiol 84 (2015) 1318-1324. doi:10.1016/j.ejrad.2015.04.014. [PubMed: 25963506]

[23]. Borghammer P, Østergaard K, Cumming P, Gjedde A, Rodell A, Hall N, Chakravarty MM, A deformation-based morphometry study of patients with early-stage Parkinson's disease, Eur. J. Neurol 17 (2010) 314-320. doi:10.1111/j.1468-1331.2009.02807.x. [PubMed: 19912319]

[24]. Marek K, Jennings D, Lasch S, Siderowf A, Tanner C, Simuni T, Coffey C, Kieburtz K, Flagg E, Chowdhury S, Poewe W, Mollenhauer B, Sherer T, Frasier M, Meunier C, Rudolph A, Casaceli C, Seibyl J, Mendick S, Schuff N, Zhang Y, Toga A, Crawford K, Ansbach A, de Blasio P, Piovella M, Trojanowski J, Shaw L, Singleton A, Hawkins K, Eberling J, Russell D, Leary L, Factor S, Sommerfeld B, Hogarth P, Pighetti E, Williams K, Standaert D, Guthrie S, Hauser R, Delgado H, Jankovic J, Hunter C, Stern M, Tran B, Leverenz J, Baca M, Frank S, Thomas CA, Richard I, Deeley C, Rees L, Sprenger F, Lang E, Shill H, Obradov S, Fernandez H, Winters A, Berg D, Gauss K, Galasko D, Fontaine D, Mari Z, Gerstenhaber M, Brooks D, Malloy S, Barone P, Longo K, Comery T, Ravina B, Grachev I, Gallagher K, Collins M, Widnell KL, Ostrowizki S, Fontoura P, La-Roche FH, Ho T, Luthman J, van der Brug M, Reith AD, Taylor P, The Parkinson Progression Marker Initiative (PPMI), Prog. Neurobiol 95 (2011) 629-635. doi:10.1016/ j.pneurobio.2011.09.005. [PubMed: 21930184]

[25]. Litvan I, Goldman JG, Tröster AI, Schmand BA, Weintraub D, Petersen RC, Mollenhauer B, Adler CH, Marder K, Williams-Gray CH, Aarsland D, Kulisevsky J, Rodriguez-Oroz MC, Burn DJ, Barker RA, Emre M, Diagnostic criteria for mild cognitive impairment in Parkinson's disease: Movement Disorder Society Task Force guidelines, Mov. Disord 27 (2012) 349-356. doi:10.1002/mds.24893. [PubMed: 22275317]

[26]. Gaser C, Nenadic I, Buchsbaum BR, a Hazlett E, Buchsbaum MS, Deformation-based morphometry and its relation to conventional volumetry of brain lateral ventricles in MRI., Neuroimage. 13 (2001) 1140-1145. doi:10.1006/nimg.2001.0771. [PubMed: 11352619]

[27]. Cabeza R, Nyberg L, Imaging cognition II: An empirical review of 275 PET and fMRI studies, J. Cogn. Neurosci (2000). doi:10.1162/08989290051137585.

[28]. Visser M, Jefferies E, Lambon Ralph MA, Semantic Processing in the Anterior Temporal Lobes: A Meta-analysis of the Functional Neuroimaging Literature, J. Cogn. Neurosci 22 (2010) 10831094. doi:10.1162/jocn.2009.21309. [PubMed: 19583477] 
[29]. Danielmeier C, Eichele T, Forstmann BU, Tittgemeyer M, Ullsperger M, Posterior medial frontal cortex activity predicts post-error adaptations in task-related visual and motor areas., J. Neurosci 31 (2011) 1780-9. doi:10.1523/JNEUROSCI.4299-10.2011. [PubMed: 21289188]

[30]. Chang LJ, Yarkoni T, Khaw MW, Sanfey AG, Decoding the Role of the Insula in Human Cognition: Functional Parcellation and Large-Scale Reverse Inference, Cereb. Cortex 23 (2013) 739-749. doi:10.1093/cercor/bhs065. [PubMed: 22437053]

[31]. Biesbroek JM, van Zandvoort MJE, Kuijf HJ, Weaver NA, Kappelle LJ, Vos PC, Velthuis BK, Biessels GJ, Postma A, The anatomy of visuospatial construction revealed by lesion-symptom mapping, Neuropsychologia. 62 (2014) 68-76. doi:10.1016/j.neuropsychologia.2014.07.013. [PubMed: 25062545]

[32]. Caproni S, Muti M, Di Renzo A, Principi M, Caputo N, Calabresi P, Tambasco N, Subclinical visuospatial impairment in Parkinson's disease: The role of basal ganglia and limbic system, Front. Neurol 58 (2014). doi:10.3389/fneur.2014.00152.

[33]. Xu X, Guan X, Guo T, Zeng Q, Ye R, Wang J, Zhong J, Xuan M, Gu Q, Huang P, Pu J, Zhang B, Zhang M, Brain Atrophy and Reorganization of Structural Network in Parkinson's Disease With Hemiparkinsonism., Front. Hum. Neurosci 12 (2018) 117. doi:10.3389/fnhum.2018.00117. [PubMed: 29636671]

[34]. Garcia-Diaz AI, Segura B, Baggio HC, Uribe C, Campabadal A, Abos A, Marti MJ, Valldeoriola F, Compta Y, Bargallo N, Junque C, Cortical thinning correlates of changes in visuospatial and visuoperceptual performance in Parkinson's disease: A 4-year follow-up, Parkinsonism Relat. Disord 46 (2018) 62-68. doi:10.1016/J.PARKRELDIS.2017.11.003. [PubMed: 29132765]

[35]. Umarova RM, Saur D, Schnell S, Kaller CP, Vry M-S, Glauche V, Rijntjes M, Hennig J, Kiselev V, Weiller C, Structural Connectivity for Visuospatial Attention: Significance of Ventral Pathways, Cereb. Cortex 20 (2010) 121-129. doi:10.1093/cercor/bhp086. [PubMed: 19406904]

[36]. Tomasino B, Gremese M, The Cognitive Side of M1., Front. Hum. Neurosci 10 (2016) 298. doi: 10.3389/fnhum.2016.00298. [PubMed: 27378891]

[37]. Kaas AL, Van Mier H, Goebel R, The neural correlates of human working memory for haptically explored object orientations, Cereb. Cortex (2007). doi:10.1093/cercor/bhl074.

[38]. Zhang J, Zhang Y-T, Hu W-D, Li L, Liu G-Y, Bai Y-P, Gray matter atrophy in patients with Parkinson's disease and those with mild cognitive impairment: a voxel-based morphometry study., Int. J. Clin. Exp. Med 8 (2015) 15383-92. http://www.ncbi.nlm.nih.gov/pubmed/ 26629027 (accessed October 1, 2018). [PubMed: 26629027]

[39]. Hogue O, Fernandez HH, Floden DP, Predicting early cognitive decline in newly-diagnosed Parkinson's patients: A practical model, Parkinsonism Relat. Disord (2018). doi:10.1016/ j.parkreldis.2018.06.031.

[40]. Serrien DJ, Sovijärvi-Spapé MM, Hemispheric asymmetries and the control of motor sequences, Behav. Brain Res. 283 (2015) 30-36. doi:10.1016/j.bbr.2015.01.021. [PubMed: 25617529] 


\section{Highlights}

- Distinct atrophy patterns are associated with different cognitive classes.

- Atrophy can be found before the development of cognitive decline.

- Longitudinal studies can determine whether these atrophy patterns affect cognition 


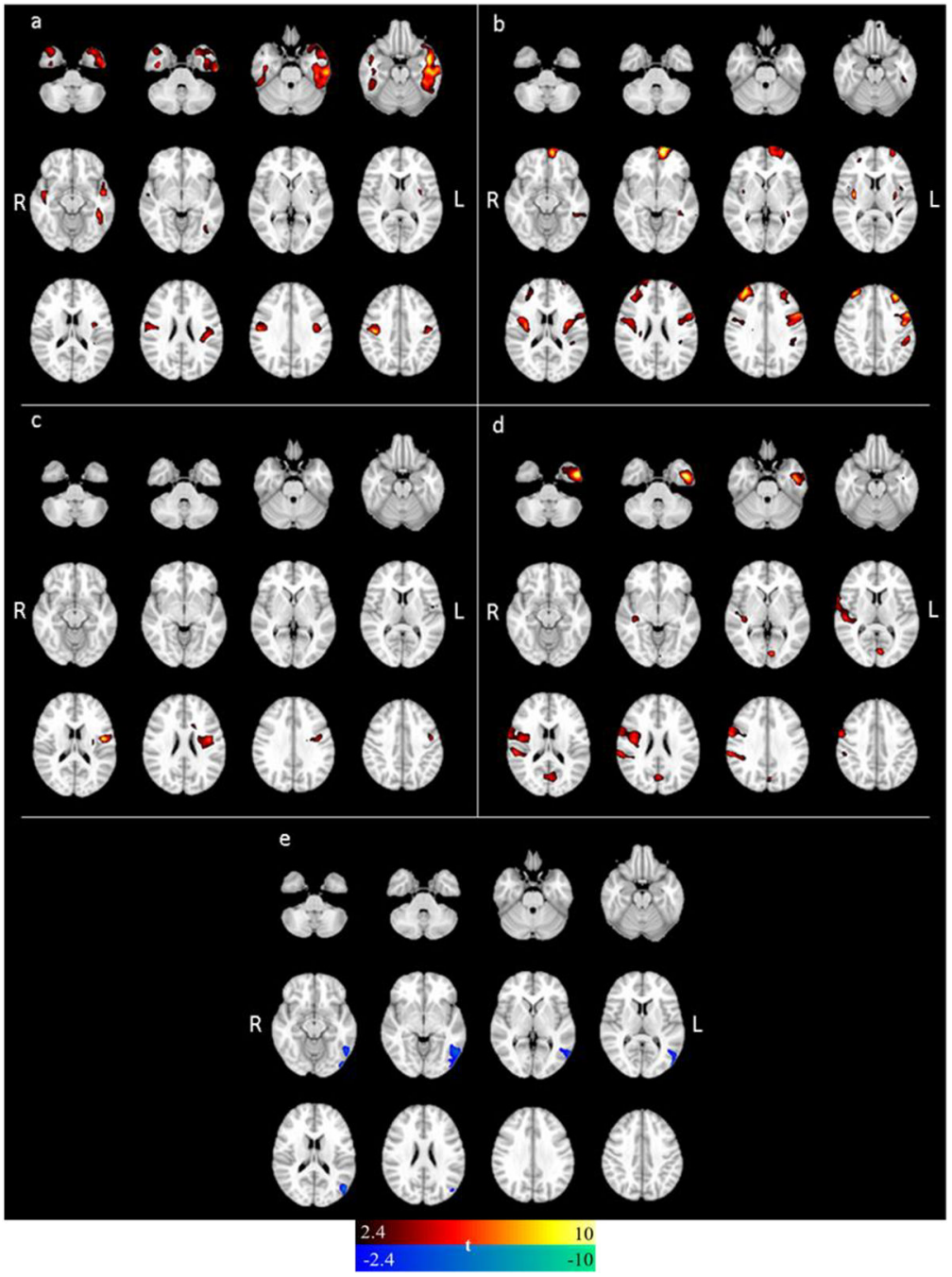

Figure 1.

Local clusters of atrophy in the a) Weak class, b) Weak-Visuospatial/Strong-Memory class, c) Weak-Visuospatial class, d) Amnestic class, e) Strong class. All comparisons were done against the Typical class. Color bar indicates the amount of difference between the two classes, a greater $t$ value indicates a larger difference. 


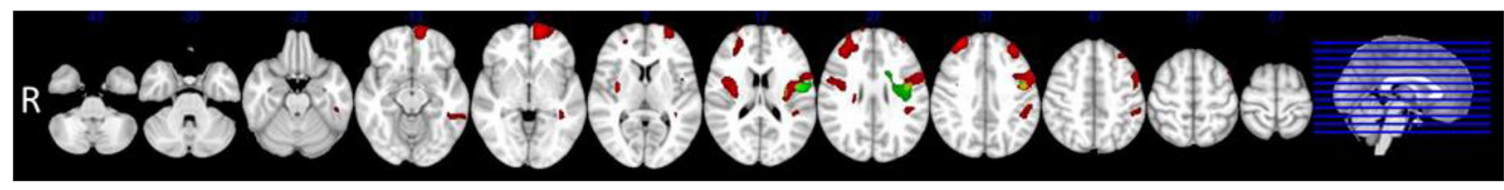

Figure 2.

Overlap of atrophy between Weak-Visuospatial/Strong Memory and the Weak-Visuospatial class. Red color indicates the atrophy in the Weak-Visuospatial/Strong-Memory class compared to the Typical class; green color indicates the atrophy in the Weak-Visuospatial class compared to the Typical class; yellow color indicates the atrophy overlap between these two classes. 


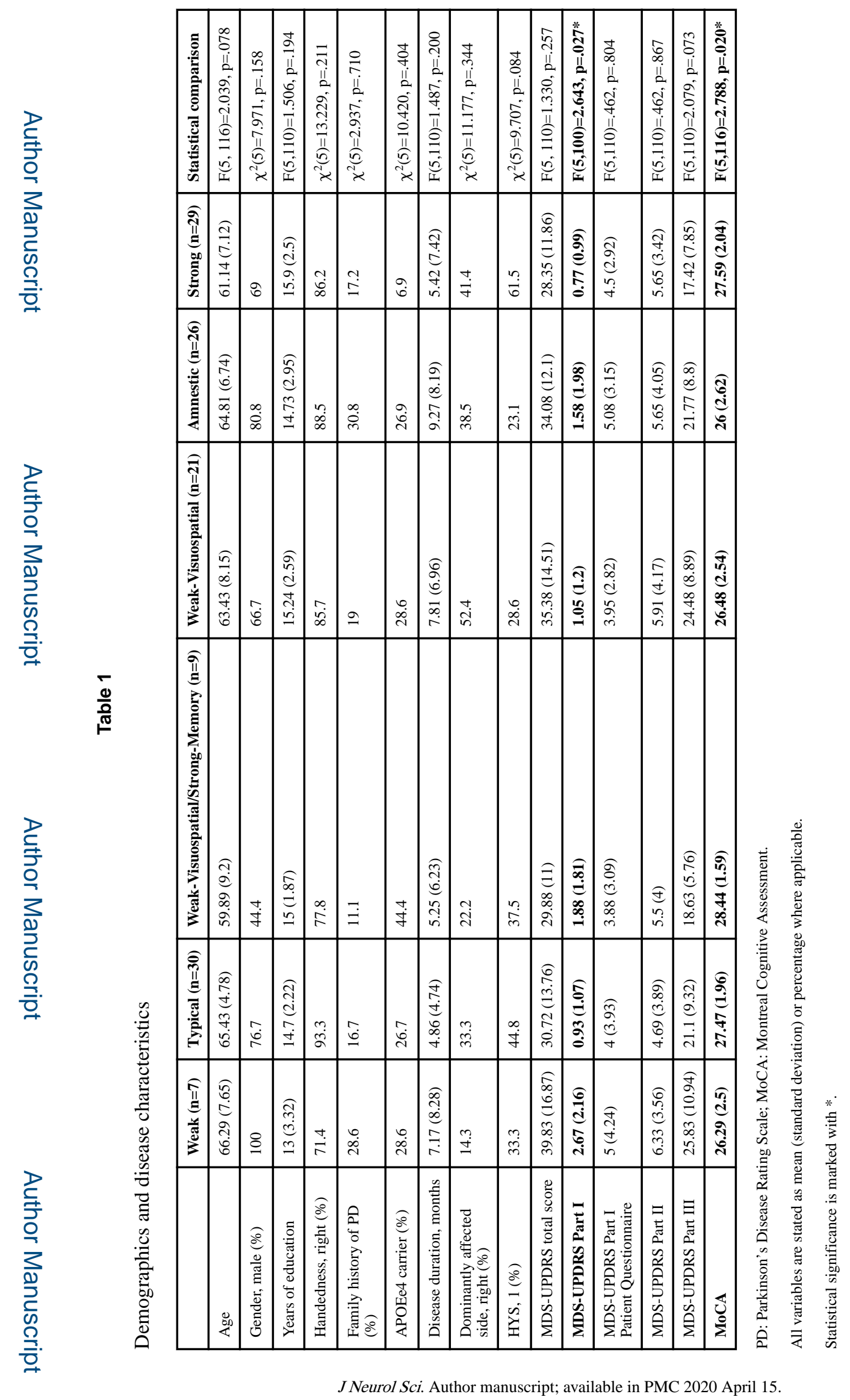




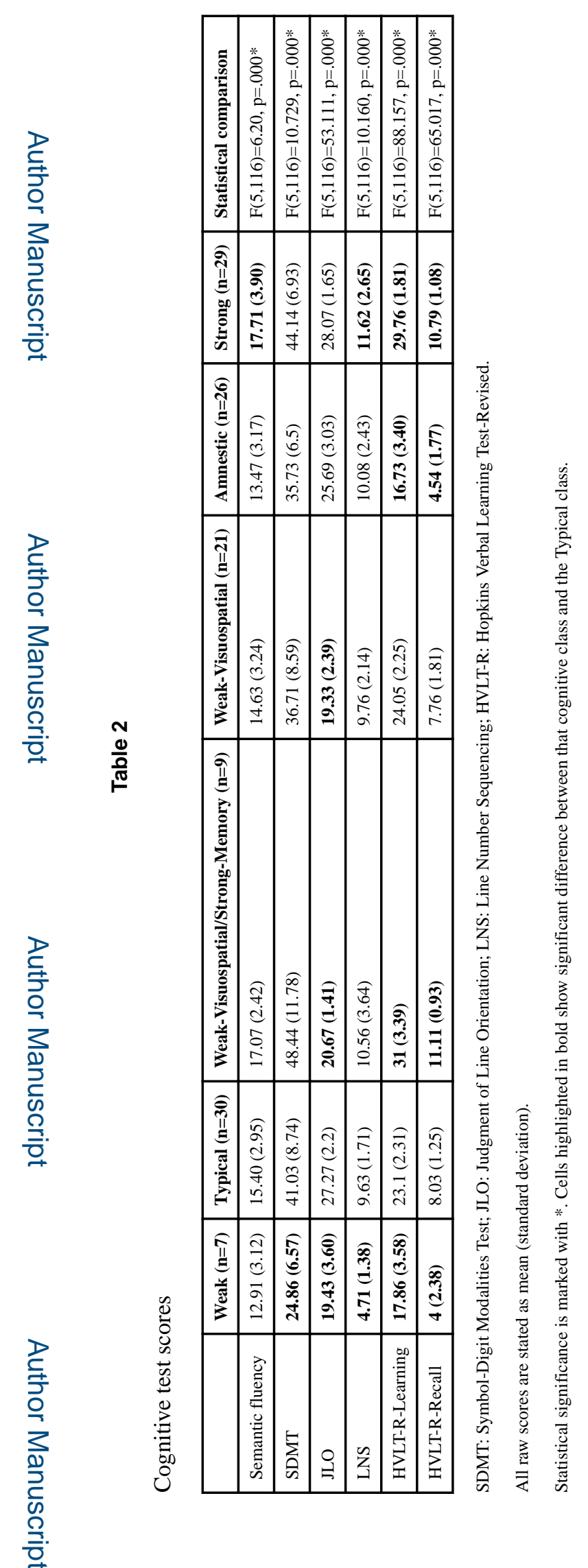

J Neurol Sci. Author manuscript; available in PMC 2020 April 15. 
Table 3

Significant clusters of atrophy in each class compared to the Typical class

\begin{tabular}{|c|c|c|c|c|c|}
\hline \multirow{2}{*}{ Region } & \multirow[b]{2}{*}{ Extent (Number of voxels) } & \multirow[b]{2}{*}{ t-value } & \multicolumn{3}{|c|}{ MNI coordinates } \\
\hline & & & $\mathbf{x}$ & $\mathbf{y}$ & $\mathbf{z}$ \\
\hline \multicolumn{6}{|c|}{ Weak } \\
\hline L Middle Temporal Gyrus & 10030 & 4.778 & -48 & 2 & -19 \\
\hline L Inferior Temporal Gyrus & 10030 & 4.498 & -44 & -39 & -16 \\
\hline R Precentral Gyrus & 4366 & 4.393 & 42 & -18 & 41 \\
\hline R Posteromedial Frontal Cortex & 4366 & 4.104 & 6 & 5 & 59 \\
\hline R Inferior Temporal Gyrus & 3063 & 3.619 & 39 & -9 & -34 \\
\hline L Rolandic Operculum & 1647 & 3.561 & -45 & -22 & 23 \\
\hline L Insular Lobe & 1647 & 3.245 & -35 & -6 & 15 \\
\hline \multicolumn{6}{|c|}{ Weak Visuospatial/Strong-Memory } \\
\hline L Superior Orbital Gyrus & 3676 & 4.683 & -12 & 62 & -9 \\
\hline L Middle Frontal Gyrus & 3676 & 4.501 & -33 & 41 & 38 \\
\hline R Middle Frontal Gyrus & 2934 & 4.549 & 33 & 48 & 35 \\
\hline R Superior Frontal Gyrus & 2934 & 2.735 & 25 & 50 & 8 \\
\hline R Putamen & 2286 & 4.482 & 34 & -9 & 11 \\
\hline R Precentral Gyrus & 2286 & 3.073 & 54 & 0 & 26 \\
\hline L Inferior Frontal Gyrus (pars opercularis) & 4122 & 4.249 & -56 & 11 & 38 \\
\hline L Insular Lobe & 4122 & 4.001 & -33 & -6 & 15 \\
\hline L Middle Frontal Gyrus & 4122 & 3.408 & -45 & 12 & 56 \\
\hline LSupramarginal Gyrus & 1890 & 3.360 & -54 & -31 & 39 \\
\hline L Inferior Temporal Gyrus & 1890 & 2.783 & -63 & -42 & -12 \\
\hline \multicolumn{6}{|c|}{ Weak-Visuospatial } \\
\hline L Rolandic Operculum & 2458 & 4.520 & -48 & -6 & 17 \\
\hline \multicolumn{6}{|c|}{ Amnestic } \\
\hline L Inferior Temporal Gyrus & 3277 & 4.955 & -45 & -3 & -37 \\
\hline L Lingual Gyrus & 1681 & 3.866 & -6 & -79 & 6 \\
\hline R Supramarginal Gyrus & 7944 & 3.788 & 61 & -31 & 29 \\
\hline R Precentral Gyrus & 7944 & 3.743 & 58 & 0 & 36 \\
\hline R Insular Lobe & 7944 & 3.666 & 37 & -6 & 21 \\
\hline \multicolumn{6}{|c|}{ Strong* } \\
\hline L Middle Temporal Gyrus & 2548 & -3.735 & -50 & -72 & 18 \\
\hline L Inferior Temporal Gyrus & 2548 & -3.393 & -56 & -61 & -7 \\
\hline
\end{tabular}

L: left; R: right.

* Atrophy was found in the Typical class when compared to the Strong class. 